\title{
Application of a double-enrichment procedure for microsatellite isolation and the use of tailed primers for high throughput genotyping
}

\author{
Fábio Mendonça Diniz ${ }^{1,2}$, Arati Iyengar ${ }^{2,3}$, Paulo Sarmanho da Costa Lima ${ }^{1}$, Norman Maclean ${ }^{2}$ \\ and Paul Bentzen ${ }^{4}$ \\ ${ }^{1}$ Embrapa Meio-Norte, Teresina, PI, Brazil. \\ ${ }^{2}$ School of Biological Sciences, University of Southampton, Southampton, UK. \\ ${ }^{3}$ Department of Forensic and Investigative Sciences, University of Central Lancashire, Preston, UK. \\ ${ }^{4}$ Department of Biology, Dalhousie University, Halifax, NS, Canada.
}

\begin{abstract}
The number of microsatellite loci and their allelic diversity contribute to increase accuracy and informativity of genetic estimates, however, the isolation of microsatellite loci is not only laborious but also quite expensive. We used $(\text { GATA })_{n}$ and $(\text { GACA })_{n}$ tetranucleotide probes and single- and double-enrichment hybridization to construct and screen a genomic library with an increased proportion of DNA fragments containing repeat motifs. Repeats were found using both types of hybridization but the double-enrichment procedure recovered sequences of which $100 \%$ contained (GATA) ${ }_{n}$ and (GACA) ${ }_{n}$ motifs. Microsatellite loci primers were then designed with an M13R-tail or CAG-tag to produce scorable PCR products with minimal stutter. The approach used in this study suggests that double-enrichment is a worthwhile strategy when isolating repeat motifs from eukaryotic genomes. Moreover, the use of tailed microsatellite primers provides increased resolution for compound microsatellite loci, with a significant decrease in costs.
\end{abstract}

Key words: double-enrichment, double-hybridization, stutter bands, tailed microsatellite primers.

Received: July 7, 2006; Accepted: October 24, 2006.

Microsatellites, also known as simple sequence repeats (SSRs), are present throughout the eukaryotic genome, often at high concentrations. They are useful markers for a wide range of analyses because in many taxa they show high levels of intraspecific genetic variability. These markers are also easily detectable by the polymerase chain reaction (PCR), producing highly reproducible results when compared with other molecular markers (Tautz et al., 1986; Wright \& Bentzen, 1994; O'Connell \& Wright, 1997). Applications of microsatellite markers include biomedical diagnosis of diseases (Girardet et al., 2005), genome mapping (Park et al., 2005), parentage analysis and relatedness of individuals or groups (Porta et al., 2006), assessing demographic history (Jacobsen et al., 2005) and examining the genetic structure of subpopulations and populations (Diniz et al., 2004). The information revealed by the genetic data obtained from these markers is a function of the number of loci and allelic diversity, both of which contribute interactively to increase the accuracy and suc-

Send correspondence to Fábio M. Diniz. Embrapa Meio-Norte, Av. Duque de Caxias 5650, Caixa Postal 01, 64006-220 Teresina, PI, Brazil. E-mail: fmd1@cpamn.embrapa.br. cess of genetic estimates and consequently the reliability of inferences (Brookfield \& Parkin, 1993; Chakraborty \& Jin, 1993; Blouin et al., 1996). However, the isolation of microsatellite loci is not only demanding but also expensive because of the need for numerous polymorphic microsatellites and, consequently, specific fluorescent primers. Many techniques have been applied (Zane et al., 2002; Fujishima-Kanaya et al., 2003; Waldbieser et al., 2003; Chen et al., 2005) but there is no guarantee that a particular method will result in a large number of loci being identified. In addition, current mainstream analysis methods involve the use of fluorescently labeled primers which are expensive, especially when large numbers of loci are required.

In an effort to increase the proportion of genomic DNA fragments containing repeat motifs a simple and inexpensive methodology is described and optimized for the construction of an enriched genomic library. Moreover, one of the primers from every pair designed for the microsatellite flanking sequences were M13-tailed or CAGtagged to produce microsatellite primer sets that were easier to score and did not require expensive fluorescent labeling. 
The spiny lobster Panulirus argus was used as a model organism in this study. Specimens were collected along the Brazilian coastline between latitudes $03^{\circ} 43^{\prime}$ and $03^{\circ} 54^{\prime}$ 'South. Samples were taken from the fresh tissue of the walking legs of the lobsters and immediately preserved in $20 \%(\mathrm{v} / \mathrm{v})$ dimethyl sulfoxide (DMSO) in saturated sodium chloride solution (Dawson et al., 1998). Genomic DNA was extracted from the tissue using the phenol/chloroform-isoamyl alcohol protocol described by Sambrook et al. (1989).

Two genomic libraries enriched for tetranucleotide repeats were prepared as outlined in Hamilton et al. (1999) and McPherson et al. (2001), with minor modifications (Diniz et al., 2005). The first library was single-enriched for $(\mathrm{GACA})_{4}$ and (GATA) $)_{7}$. The second library was constructed using an additional enrichment after the first hybridization capture (biotin/streptavidin) technique (Kijas et al., 1994) with the previously used tetranucleotide probes. Genomic DNA ( $200 \mathrm{ng} / \mu \mathrm{L})$ was digested separately with HincII, RsaI, Bst $\mathrm{UI}$ and HaeIII at $37^{\circ} \mathrm{C}$ overnight. Digests were recovered using PCR Purification columns (Qiagen) according to manufacturer's instructions, and the terminal ends dephosphorylated using calf intestinal phosphatase. The digested-dephosphorylated genomic DNA was cleaned of all modifying enzymes and then ligated to doublestranded linkers, termed SNX ( $\underline{S} u \mathrm{I}, \underline{N} h e \mathrm{I}$ and $\underline{X} m n \mathrm{I})$ for the restriction sites they contain or form when dimerisation occurs (SNX-F: 5'-CTAAGGCCTTGCTAGCAGAAGC-3' and SNX-R: 5'-pGCTTCTGCTAGCAAGGCCTTAGAA AA-3'). The ligation reaction used the enzyme $T_{4}$ DNA ligase in the presence of $X m n \mathrm{I}$ to prevent blunt-ended dimers between complementary linkers. Linker-ligated fragments were PCR amplified with the SNX-F linker (Hamilton et al., 1999) as primer. Amplifications were carried out in a $50 \mu \mathrm{L}$ reaction volume containing $20-100 \mathrm{ng}$ DNA, $1 \times$ Thermopol ${ }^{\circledR}$ buffer supplemented with $1.5 \mathrm{mM}$ $\mathrm{MgCl}, 50 \mu \mathrm{M}$ of each dNTP, $0.5 \mathrm{U}$ Taq DNA polymerase, and 0.3 to $0.5 \mu \mathrm{M}$ of SNX-F linker. The PCR amplifications were carried out on a MJ Research DNA Engine Tetrad PTC-225 thermalcycler using the following protocol: $95^{\circ} \mathrm{C}$ for $5 \mathrm{~min}$, followed by 20 cycles $\left(95^{\circ} \mathrm{C}\right.$ for $45 \mathrm{~s}$, $62{ }^{\circ} \mathrm{C}$ for $1 \mathrm{~min}$ and $72{ }^{\circ} \mathrm{C}$ for $2 \mathrm{~min}$ ) and then $72{ }^{\circ} \mathrm{C}$ for $30 \mathrm{~min}$. Unless otherwise stated, all materials came from New England Biolabs, USA.

The PCR products were used for subtractive hybridization with biotinylated tetranucleotide probes (GACA) ${ }_{4}$ and (GATA $)_{7}$ (Operon Technologies) bound to magnetic beads (Dynal Biotech Inc.). Following hybridization, the beads were washed with saline sodium citrate buffer in the presence of $0.5 \mathrm{ng} \mu \mathrm{L}^{-1}$ of SNX-F primer. After the final wash, $30 \mu \mathrm{L}$ of Tris-EDTA buffer $(10 \mathrm{mM}$ Tris, $0.1 \mathrm{mM}$ EDTA, $\mathrm{pH}$ 8.0) was added to the beads, and the mixture was incubated at $98^{\circ} \mathrm{C}$ for $15 \mathrm{~min}$ to release the DNA from the probes. The enriched-DNA recovered from the beads was amplified again with the SNX-F linker to generate double stranded DNA using the PCR conditions described above. Amplified-enriched DNA was cleaned with purification columns (Qiagen) and a second round of hybridization was performed using a small fraction of the postenrichment amplified inserts and the same tetranucleotide probes and hybridization conditions described above. Double-enriched DNA was recovered from the beads and PCR amplified once again under the same thermal profile as first described, except for variation in the number of cycles, between 10 and 25.

Amplified-enriched DNA from the single- and double-enrichment procedures was ligated into the pDrive cloning vector (Qiagen PCR cloning kit) and used to transform OneShot ${ }^{\circledR}$ competent Escherichia coli (Invitrogen) which were plated onto Lauria-Bertani agar (LB; Difco) supplemented with $50 \mu \mathrm{g} \mathrm{mL}^{-1}$ ampicillin. Plasmid inserts were amplified directly from the transformed $E$. coli colonies (PCR screening) using primers (M13-F: 5'-GGAA ACAGCTATGACCATG-3' and M13-R: 5'-GTAAAAC GACGCCAGTG-3') that flank the cloning site and sizefractionated on $1.5 \%(\mathrm{w} / \mathrm{v})$ agarose gels. Recombinant $E$. coli clones giving a positive signal for the insertion of the $P$. argus 500-1000 base-pair (bp) DNA sequence were incubated overnight on LB-ampicillin and the plasmid DNA purified and extracted using the Qiaprep ${ }^{\circledR}$ spin miniprep kit (Qiagen). Each clone was cycle-sequenced $\left(96^{\circ} \mathrm{C}\right.$ for $3 \mathrm{~min}$, followed by 40 cycles of $96^{\circ} \mathrm{C}$ for $20 \mathrm{~s}, 52^{\circ} \mathrm{C}$ for $20 \mathrm{~s}$ and $60^{\circ} \mathrm{C}$ for $4 \mathrm{~min}$ ) in one direction using the M13-F primer and the CEQ DTCS - Quick Start kit (Beckman Coulter Inc.) and sequenced on a CEQ 8000XL DNA Analysis System (Beckman Coulter Inc.). Confirmed positives were further sequenced for the opposite strand using the SP6 (5'-CATACGATTTAGGTGACACTATAG-3') and M13-R universal primers.

Microsatellite loci primers were designed on the unique flanking regions of each locus using PRIMER 3 (Rozen \& Skaletsky, 2000) and an oligonucleotide tail corresponding to the 5'-GGAAACAGCTATGACCAT-3' M13-R universal primer (Oetting et al., 1995; BoutinGanache et al., 2001) or the 5'-CAGTCGGGCGTCATC A-3' CAG tag (Hauswaldt \& Glenn, 2003) was added to the 5 ' end of one primer of each pair to facilitate fluorescent labeling of the PCR products during amplification. This approach allowed the amplification with three primers, a tailed STR primer conjugate (forward - $\mathrm{F}_{\mathrm{T}}$ or reverse $-\mathrm{R}_{\mathrm{T}}$ ), a non-tailed STR primer ( $\mathrm{F}$ or R) and the labeled primer containing either hexachloro-6-carboxyfluorescein (HEX) or 6-carboxyfluorescein (FAM) fluorescent dye $\left(\mathrm{T}_{\mathrm{L}}\right)$. The labeled primer was the M13R or CAG tag oligonucleotide and therefore could be used with any primer containing the same sequence as the tail. Selection of each tail - primer combination was made using the computer program GENRUNNER v 3.02 (Hastings Software, USA). 
HaeIII-digested samples produced mainly high (> $3000 \mathrm{bp}$ ) molecular weight fragments with no specific banding pattern and were subjected to further experiments. Minimum over-amplification of ligated DNA and repeatenriched fragments was warranted when PCR was carried out with 15 cycles.

The libraries enriched for tetranucleotide repeat motifs showed high levels of microsatellite enrichment (Figure 1). Each enriched library had 32 provisional positive clones sequenced. The single-enriched library showed very similar numbers of sequences containing no repeat motifs and sequences recovered in duplicate, except for the $(\text { GATA })_{7}$ library, which had four times more sequences containing no repeats compared to the library probed with (GATA) 4 (Figure 1A). Moreover, this more commonly used sequence enrichment resulted in three sequences being unsuitable for primer design because they either had short or no available flanking regions (Figure 1A). The mean percentage of sequences with repeat motifs from single-enriched libraries was high (67\%), ranging from about 9 microsatellite sequences (55\%) for (GACA $)_{4}$ to about 13 microsatellite sequences $(80 \%)$ for (GATA) 7 libraries (Figure 1B). The overall high success rate of the single-
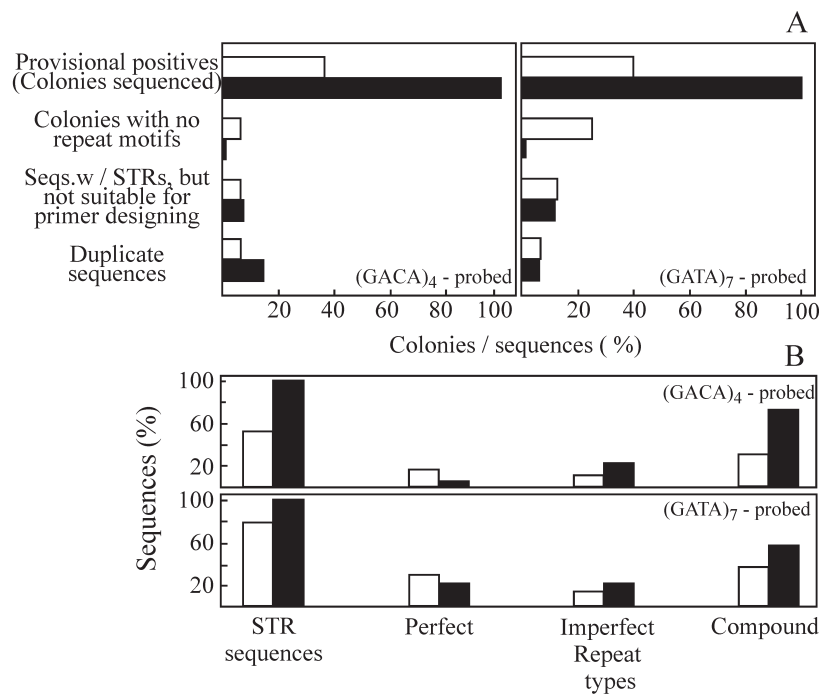

Figure 1 - Evaluation of the enrichment procedures success (A) and characterization of repeat motifs recovered from the process (B). $\square$ single-hybridization, $\mathbf{a}$ double-hybridization. enrichment protocol employed here was probably due to the presence of large numbers of these tetranucleotide repeats within the $P$. argus genome coupled with the effectiveness of using the hybridization capture (biotin/ streptavidin) enrichment technique (Kijas et al., 1994). Double-enrichment produced libraries in which all of the sequences contained (GACA $)_{n}$ and $(\text { GATA })_{n}$ motifs $(16$ sequences each). Out of the 32 sequences isolated after double-enrichment, six had limited flanking sequence for primer design or were second copy clones. The high frequency of positive clones found in the recombinant colonies also suggests that PCR screening for fragments of appropriate length (500-1000 bp inserts) considerably increases the chance of detecting clones with repeat arrays and with longer flanking regions for primer design.

Microsatellite sequences from both procedures were characterized by length and type according to Weber (1990) (Figure 1B). Most tandem arrays recovered from the single enrichment procedure were compound (11 sequences) or imperfect repeats ( 3 sequences). This was even more noticeable in the double-enriched library, where 21 microsatellite sequences were compound repeats and $7 \mathrm{se}-$ quences were imperfect repeats. Compound microsatellites were frequently associated with dinucleotides $(\mathrm{AC} / \mathrm{TG})_{\mathrm{n}}$ in the case of the GACA-enriched library and with $(\mathrm{GA} / \mathrm{CT})_{\mathrm{n}}$ in the case of the GATA-enriched library. The relative frequency of perfect repeat sequences was higher in singleenrichment, especially for the GATA repeat motif. Microsatellite sequences have been deposited in GenBank under accession numbers AY536335 to AY536353 and further characterized (including the primer sequences) by Diniz et al. $(2004,2005)$.

Genotyping 32 P. argus samples at all the microsatellite loci isolated in this study showed clear bands with no shadow bands and minimal stutter (Figure 2). Shadow or stutter bands, occurring during PCR due to replication slippage are not a common difficulty in scoring tetranucleotide loci, but may be a problem for microsatellite loci consisting of compound tetranucleotide loci (i.e. tetra + dinucleotides) producing stutter fragments to levels seen with dinucleotides (Hauge \& Litt 1993; O'Reilly et al., 2000). The tailing of one of the microsatellite primers per locus has been found to improve scoring of stuttering fragments from di-,

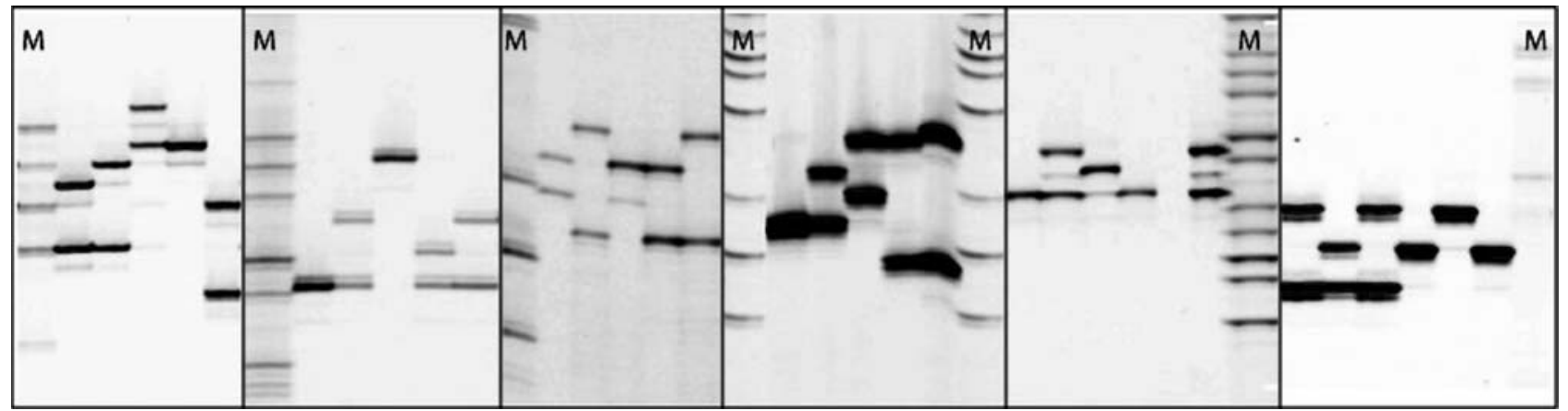

Figure 2 - An inverted tone image of a polyacrylamide gel $(6 \%, \mathrm{w} / \mathrm{v})$, showing the banding pattern of microsatellite loci amplified with tailed PCR primers. M: Molecular size marker. 
tri- or tetranucleotide microsatellite amplifications (Oetting et al., 1998; Boutin-Ganache et al., 2001). This increases the robustness of scoring, and eliminates the end-labeling of one of the PCR primers for each specific locus, thus reducing the costs associated with labeling primers with fluorescent dye (Yu et al., 2001). However, the inclusion of such tails does increase the chance of formation of hairpins and primer-dimers (Hauswaldt \& Glenn, 2003). Therefore, the selection of each tail - primer combination should be tested using a specialized program such as the GENRUNNER program to minimize potential secondary structures. Because the number of primers is increased in the PCR reaction, optimization of each primer set could be more labour-intensive. However, this is a drawback that can easily be overcome, and the benefits of the procedure far outweigh the drawbacks.

Our results show that double-enrichment should certainly be considered in order to overcome low percentages of clones containing tandem repeats. In this study, we were able to increase the number of sequences with repeat sequences to $100 \%$. Even though we did not notice any problem with recombination in the PCR following the hybridization steps, care should be taken to avoid this possible complication. Keeping PCR cycles to a minimum seems to be an effective way to overcome the problem. The use of a double-enrichment protocol and the addition of a sequence tag to the microsatellite amplification primers provide an inexpensive approach for increasing the number of loci that can be screened and decreases the costs of genotyping. We are conducting further work to apply these markers to population discrimination of $P$. argus throughout its range.

\section{Acknowledgments}

We thank Dr. Masayoshi Ogawa, from the Federal University of Ceará, for providing lobster tissue samples and Ian Paterson, from Dalhousie University, Canada, for technical assistance. Thanks are also due to the Brazilian Institute for the Environment and Natural Renewable Resources (IBAMA). FM Diniz was supported by a $\mathrm{PhD}$ scholarship from the Brazilian Government (CAPES).

\section{References}

Blouin MS, Parsons M, Lacaille V and Lotz S (1996) Use of microsatellite loci to classify individuals by relatedness. Mol Ecol 7:393-401.

Boutin-Ganache I, Raposo M, Raymond M and Deschepper CF (2001) M13-tailed primers improve the readability and usability of microsatellite analyses performed with two different allele-sizing methods. Biotechniques 31:24-28.

Brookfield JFY and Parkin DT (1993) Use of single-locus DNA probes in the establishment of relatedness in wild populations. Heredity 70:660-663.

Chakraborty R and Jin L (1993) A unified approach to study hypervariable polymorphism: Statistical considerations of determining relatedness and population distances. In: Pena SDJ, Chakraborty R, Epplen JT and Jeffreys AJ (eds) DNA
Fingerprinting: State of the Science. Birkhauser Verlag, Basel, pp. 153-175.

Chen K, Knorr C, Bornemann-Kolatzki K, Ren J, Huang L, Rohrer GA and Brenig B (2005) Targeted oligonucleotide-mediated microsatellite identification (TOMMI) from large-insert library clones. BMC Genet 6:54.

Dawson MN, Raskoff KA and Jacobs DK (1998) Field preservation of marine invertebrate tissue for DNA analyses. Mol Mar Biol Biotech 7:145-152.

Diniz FM, Maclean N, Ogawa M, Paterson I and Bentzen P (2005) Microsatellites in the overexploited spiny lobster, Panulirus argus: Isolation, characterization of loci and potential for intraspecific variability studies. Cons Gen 6:637-641.

Diniz FM, Maclean N, Paterson IG and Bentzen P (2004) Polymorphic tetranucleotide microsatellite markers in the Caribbean spiny lobster, Panulirus argus. Mol Ecol Notes 4:327-329.

Fujishima-Kanaya N, Toki D, Suzuki K, Sawazaki T, Hiraiwa H, Iida M, Hayashi T, et al. (2003) Development of 50 geneassociated microsatellite markers using BAC clones and the construction of a linkage map of swine chromosome 4 . Anim Genet 34:135-141.

Girardet A, Moncla A, Hamamah S and Claustres M (2005) Strategies for preimplantation genetic diagnosis of Angelman syndrome caused by mutations in the $U B E 3 A$ gene. Reprod Biomed Online 10:519-526.

Hamilton MB, Pincus EL, Di Fiore A and Flesher RC (1999) A universal linker and ligation procedures for construction of genomic DNA libraries enriched for microsatellites. Biotechniques 27:500-507.

Hauge XY and Litt M (1993) A study of the origin of shadow bands seen when typing dinucleotide repeat polymorphisms by PCR. Hum Mol Genet 2:411-415.

Hauswaldt JS and Glenn TC (2003) Microsatellite DNA loci from Diamondback terrapin (Malaclemys terrapin). Mol Ecol Notes 3:174-176.

Jacobsen BH, Hansen MM and Loeschcke V (2005) Microsatellite DNA analysis of northern pike (Esox lucius L.) populations: Insights into the genetic structure and demographic history of a genetically depauperate species. Biol J Linn Soc Lond 84:91-101.

Kijas JMH, Fowler JCS, Garbett CA and Thomas MR (1994) Enrichment of microsatellites from the citrus genome using biotinylated oligonucleotide sequences bound to streptavidin-coated magnetic particles. Biotechniques 16:657-662.

McPherson AA, O'Reilly PT, McParland TL, Jones MW and Bentzen P (2001) Isolation of nine novel tetranucleotide microsatellites in Atlantic herring (Clupea harengus). Mol Ecol Notes 1:31-32.

O'Connell M and Wright JM (1997) Microsatellite DNA in fishes. Rev Fish Biol Fish 7:331-363.

Oetting WS, Armstrong CM, Ronan SM, Young TL, Sellers TA and King RA (1998) Multiplexed short tandem repeat polymorphisms of the Weber 8A set of markers using tailed primers and infrared fluorescence detection. Electrophoresis 19:3079-3083.

Oetting WS, Lee HK, Flanders DJ, Wiesner GL, Sellers TA and King RA (1995) Linkage analysis with multiplexed short tandem repeat polymorphisms using infrared fluorescence and M13 tailed primers. Genomics 30:450-458. 
O'Reilly PT, Canino MF and Bentzen P (2000) Isolation of twenty low stutter di- and tetranucleotide microsatellites for population analyses of walleye pollock (Theragra chalcogramma) and other gadoids. J Fish Biol 56:10741086.

Park Y-H, Alabady MS, Ulloa M, Sickler B, Wilkins TA, Yu J, Stelly DM, et al. (2005) Genetic mapping of new cotton fiber loci using EST-derived microsatellites in an interspecific recombinant inbred line cotton population. Mol Genet Genomics 274:428-441.

Porta J, Porta JM, Martínez-Rodríguez G and Alvarez MC (2006) Genetic structure and genetic relatedness of a hatchery stock of Senegal sole (Solea senegalensis) inferred by microsatellites. Aquaculture 251:46-55.

Rozen S and Skaletsky HJ (2000) Primer3 on the WWW for general users and biologist programmers. In: Krawetz $\mathrm{S}$ and Misener S (eds) Bioinformatics Methods and Protocols: Methods in Molecular Biology. Humana Press, Totowa, pp 365-386.
Sambrook J, Fritsch EF and Maniatis T (1989) Molecular Cloning: A Laboratory Manual. Cold Spring Harbor Laboratory Press, Cold Spring Harbor, pp 6.4-6.11.

Tautz D, Trick M and Dover GA (1986) Cryptic simplicity in DNA is a major source of genetic variation. Nature 322:652-656.

Waldbieser GC, Quiniou SM and Karsi A (2003) Rapid development of gene-tagged microsatellite markers from bacterial artificial chromosome clones using anchored TAA repeat primers. Biotechniques 35:976-979.

Weber JL (1990) Informativeness of human (dC-dA)n(dG-dT) polymorphisms. Genomics 7:524-530.

Wright JM and Bentzen P (1994) Microsatellites: Genetic markers for the future. Rev Fish Biol Fish 4:384-388.

Yu K, Haffner M and Poysa V (2001) Tailed primer base excision sequence scanning (TP-BESS) for detection of single nucleotide polymorphisms (SNPs). Plant Mol Biol Rep 19:49-54.

Zane L, Bargelloni L and Patarnello T (2002) Strategies for microsatellite isolation: A review. Mol Ecol 11:1-16.

Associate Editor: Emmanuel Dias Neto 